\title{
Mundos virtuales y avatares como nuevas formas educativas
}

\author{
Miguel Ángel Poveda CRIAdo \\ UDIMA, UFV, UE Madrid - España e IE University - Segovia \\ mapoveda1@yahoo.es \\ $\mathrm{M}^{\mathrm{a}}$ del Carmen Thous Tuset. \\ Universidad Francisco de Vitoria de Madrid-España. \\ carmenthous@gmail.com
}

\section{Resumen:}

Learning-by-doing es el nuevo concepto educativo en el que las nuevas tecnologías, en el campo de los mundos virtuales y las redes, son una baza en la enseñanza universitaria a distancia dentro del marco EEES. Esta es la gran apuesta que tiene Internet en la educación, en donde Second Life es fundamental, dado su interactividad entre profesores y alumnos. Además, este mundo virtual permite a sus residentes construir sus propios ambientes y poder llevar una vida virtual. En España-UPNA, la Universidad de Alcalá de Henares o UDIMA tienen su propio espacio virtual dentro de SL.

Palabras clave: Learning-by-doing, redes, nuevas tecnologías, second life

\section{Virtual worlds and avatars as new education forms}

\begin{abstract}
:
Learning-by-doing is the new educational concept in which the new technologies in the field of virtual worlds and networks are an asset in distance university education within the EEES framework. This is the great challenge of the Internet in education, where Second Life is essential, given its interactivity between teachers and students. In addition, this virtual world allows its residents to build their own environment and to lead a virtual life. In Spain-Public University of Navarre, the University of Alcalá de Henares or UDIMA have their own virtual space in SL.
\end{abstract}

Key Words: Learning-by-doing, networkss, we technologies, second life

\section{Referencia normalizada:}

Poveda Criado, M. A.; Thous Tuset, M. C. (2013) Mundos virtuales y avatares como nuevas formas educativas. Historia y Comunicación Social. Vol. 18 No Especial Noviembre. Págs. 469-479.

Sumario: 1. Metodología. 2. Intoducción: Second Life y sus ventajas en la educación universitaria. 3. Usos educativos de Second Life. El caso de UDIMA en la enseñanza con mundos virtuales y avatares. 4. Conclusiones. 5. Bibliografía 


\section{Metodología}

El presente artículo se estructura en tres secciones:

1. Introducción: Second Life y sus ventajas en la educación universitaria.

2. Usos educativos de Second Life. El caso de UDIMA.

3. Conclusiones.

\section{Introducción: Second Life y sus ventajas en la educación universitaria.}

Second Life (SL) (http://secondlife.com) es el mundo virtual más grande de Internet con más de diez millones de usuarios registrados, de los cuales hay 90 mil conectados simultáneamente. Muy pocos sitios web tienen esa cantidad de visitantes al mismo tiempo. El entorno conectado con la economía real, fue creado por Linden Labs (http://lindenlab.com/) para proveer un ambiente de inmersión.

Los lemas de Second life son: "Your world, your imagination”, lo que significa: tu mundo, tu imaginación. Y es que, Second Life es, una plataforma a modo de lienzo en blanco en el que los jugadores pueden hacer uso de su imaginación. Treinta y dos años es la edad media de los jugadores. Respecto a ubicaciones, el 25\% de los usuarios no son estadounidenses. Por sexo, el 43\% son mujeres. Creada el 23 de Junio de 2003-, es considerada un metaverso ${ }^{1}$, es decir, un entorno en donde los humanos interactúan social y económicamente como iconos, a través de un soporte lógico en un ciberespacio que actúa como una especie de metáfora del mundo real.

Sus usuarios, mayores de 18 años, son conocidos como "residentes", y acceden a Second Life mediante el uso de uno de los múltiples programas de interfaz llamados viewers (visores), los cuales les permiten interactuar entre ellos mediante la creación de un ávatar ${ }^{2}$. Los residentes pueden así explorar este mundo, interactuar con otros residentes, establecer relaciones, participar en actividades individuales, como en grupo y crear y comerciar propiedad virtual y servicios. Esto supone que este mundo permite, construir y crear a sus residentes sus propios ambientes para disponer de todo lo necesario para poder llevar una vida virtual en la que, como en la normal, la enseñanza juega un papel clave.

Second Life es un juego masivo online que puede ser usado de modo gratuito y cuya apertura estructural lo hace atractivo como lugar virtual para la enseñanza. Por

1 El término metaverso (del inglés metaverse, contracción de meta universe, o sea de meta-universo) tiene su origen en la novela Snow Crash publicada en 1992 por Neal Stephenson, y se usa frecuentemente para describir una visión de trabajo en espacios 3D. En definitiva, un metaverso es el mundo virtual ficticio descrito en la citada obra Snow Crash («Samurai virtual”)

2 Avatar es un término hindú que se refiere a la encarnación terrestre de un dios. En idioma español también significa 'vicisitud' («los avatares de la vida»). 
esta razón, las universidades de todo el mundo han creado su espacio dentro de SL con el objetivo de que sus estudiantes puedan acceder al proceso de enseñanza-aprendizaje desarrollado en cada uno de sus estudios. Con estas actividades se refuerzan y solidifican los conocimientos del estudiante a partir del método Learning-by-doing.

SL es una plataforma que, aprovechando el atractivo de los entornos de inmersión tridimensionales y la telepresencia en forma de avatares, ofrece posibilidades interesantes para que el profesor universitario lo utilice como vehículo complementario o incluso, único de su enseñanza virtual. Como señalan Daniel Livingstone y Jeremy Kemp (2006), la interfaz tridimensional de SL resulta mucho más alegre para estudiantes que encuentran aburridas las plataformas de aprendizaje virtual (tales como WebCT y Moodle) porque sólo ofrecen entornos bidimensionales. Según Linden $\mathrm{Lab}^{3}$.

Second Life ofrece un entorno único y flexible para los educadores interesados en la enseñanza a distancia, el trabajo cooperativo por medio del ordenador, la simulación, los estudios de new media y la formación empresarial.

Desde que los seres humanos creyeron en los mitos y leyendas "la virtualidad se hizo carne y habitó entre nosotros. Los entes virtuales han formado parte de la vida social en todas las culturas" (Echeverría, 2000, p.23-24). El comienzo de estos mundos virtuales entre los que se encuentra Second Life, nos lleva al mundo de los videojuegos, que fueron los primeros que crearon nuevos mundos copia del real. Hasta los 90, la educación no contó con los nuevos mundos virtuales. Las primeras universidades que comienzan interesarse son las de Carolina del Norte y California en Estados Unidos. Second Life utiliza la metáfora que MacLuhan "el aula sin muros", facilitando la docencia online, con nuevos modelos más visuales.

Este entorno está abierto a todo tipo de iniciativas, una de ellas es que la de ofrecer educación de calidad en las artes y las ciencias para todos los ciudadanos de Second Life. Según Edward Castronova (2001), especialista en mundos sintéticos, hay tres características fundamentales de Second Life y de los metaversos: Interactividad, corporeidad y persistencia.

La interactividad se refiere a que el usuario es capaz de comunicarse con el resto de usuarios y de interactuar y ejercer influencia. En plataformas únicamente textuales es difícil coordinar chats con más de 10 alumnos a la vez. Second Life, gracias a su alto grado de interactividad y la presencia visual de los participantes (se ve cuándo hablan porque se los ve teclear y aparece un sonido de teclas), resulta más ágil.

3 Empresa que desarrolló un programa que permitía a sus usuarios la inmersión en un mundo virtual. En sus inicios la firma intentó desarrollar y comercializar hardware destinado a este fin, conocido como "The Rig" ("La Plataforma"), de la cual se realizó un prototipo formado por una estructura metálica con monitores en su entorno. De esa visión se pasó a la aplicación conocida como Linden World, en la cual diferentes personas podían participar en juegos orientados a tareas específicas, al igual que socializar en un entorno tridimensional on-line. «Linden Lab To Open Source Second Life Software». Linden Lab Consultado el 3 de agosto de 2013. 
La corporeidad es que los usuarios están representados por avatares, limitados por una altura y un peso, en un espacio que también posee ciertos límites y está sometido a ciertas leyes.

Por último, la persistencia significa que el programa sigue funcionando y desarrollándose a pesar de que los miembros no estén conectados. Además, las posiciones en las que se encontraban los usuarios al cerrar sus sesiones, así como sus conversaciones, objetos de propiedad, etc., siempre son guardadas, lo que permite recuperarlos cuando nos volvamos a conectar.

Estas características convierten a SL en un aprendizaje por inmersión: aprender haciendo. La palabra inmersión nos recuerda al vocabulario marino, y más concretamente al acto por medio del cual el hombre entra en el mar con el fin de desarrollar una actividad. El Diccionario de la Real Academia ${ }^{4}$ define el término en su primera acepción como la "acción de introducir o introducirse algo en un fluido. La experiencia de inmersión puede definirse también como una experiencia de la atención. Lo que hace aún más interesante este mundo es que una vez que abrimos una cuenta y accedemos a él, todo se convierte en aprendizaje: tienes que aprender a crearte, aprender a moverte, a construir, interactuar, socializarte, etc. En SL "aprender es hacer" y el Learning-by-doing que reclamaba Downes. En Second Life la paradoja es que, como dice Roger Schank, "el aprendizaje ocurre cuando alguien quiere aprender, no cuando alguien quiere enseñar". Este pensador defiende que las escuelas y las universidades son inútiles y que la única forma de aprender es haciendo cosas que sirvan para algo.

El profesor, en SL, debe ejercer de mentor, es decir, no es el guardián de todo el conocimiento, pero ayuda al alumno y le guía.

Entre otras ventajas de esta plataforma, podemos señalar que puede reunir a distintos grupos de estudiantes sin la necesidad de desplazarse a un aula física, permite incorporar contenidos de aprendizaje en distintos formatos (vídeo, textos, fotos, etc.), así como grabar esos contenidos y expandirlos a otras plataformas web. Los contenidos se ofrecen en un formato dominado por la imagen tridimensional y la carga visual es mucho más fuerte que la textual. También permite el aprendizaje, creación y exploración de modelos tridimensionales. Relacionado con la imagen tridimensional, está la posibilidad de explorar toda clase de cuerpos y objetos desde puntos de vista completamente innovadores y meternos en el interior de los elementos, lo que supone una aplicación educativa muy interesante. Hay que señalar como ventaja que el alumno es el protagonista, adoptando un rol activo a través de la manipulación de su avatar. En SL, la función del docente es la de animador, facilitador, organizador o guía que acompaña al alumno en su exploración, y no la de alguien que entrega el contenido o el único que explica y transmite el funcionamiento de algo.

Respecto a las desventajas como plataforma educativa, su limitación más evidente es que no es accesible a todo el mundo. También, podemos encontrar problemas

4 http://www.rae.es/rae.html 
tecnológicos. Por otro lado, el lenguaje corporal es limitado. Otro punto a tener en cuenta es el inconveniente del lag, que es el tiempo que pasa desde que un usuario usa su teclado o ratón para hacer algo hasta que esa acción aparece representada en la pantalla por medio de su avatar. De hecho, cuando concurren un grupo amplio de estudiantes tiende a producirse, lo que complica las prácticas de enseñanza y aprendizaje. Otro problema directamente relacionado es la cacofonía.

No obstante, las posibilidades educativas de SL son originales, distintas y enriquecedoras. Como ejemplo, el 25 de mayo de 2007, 1.300 educadores de todo el mundo se encontraron en la conferencia internacional: "Second Life Best Practices in Education: Teaching, Learning, and Research 2007" durante 24 horas ininterrumpidas. Fue la primera conferencia sobre educación celebrada únicamente en un mundo virtual y, tanto educadores como expertos en e-learning de todo el mundo, pudieron participar en las 30 conferencias que tuvieron lugar y debatir sobre más de 100 temas relacionados con la formación y el aprendizaje en Second Life.

Muchas universidades y empresas de todo el mundo están utilizando Second Life para la formación. En España (4 universidades en red: Alcalá de Henares, Vigo, Salamanca y UDIMA) ${ }^{5}$, tienen creadas sus propias islas y sus entornos virtuales.

Para la educación trabajar con un soporte como Second Life es útil porque, como explica Linden Lab, "Second Life ofrece un entorno único y flexible para los educadores interesados en la enseñanza a distancia, el trabajo cooperativo por medio del ordenador, la simulación, los estudios de new media y la formación empresarial"6.

Según Ana Eva Iribas?, "Second Life ofrece la oportunidad de usar la simulación en un entorno seguro para acrecentar el aprendizaje experiencial, permitiendo a los individuos practicar habilidades, probar nuevas ideas y aprender de sus errores. ¡ $\mathrm{La}$ capacidad de prepararse para experiencias similares en el mundo real, usando Second Life como simulación, tiene un potencial ilimitado!".

Estudiantes y educadores de cualquier parte del mundo pueden trabajar juntos en Second Life en un aula virtual en red global. El uso de esta herramienta como un complemento a los entornos tradicionales del aula también ofrece nuevas oportunidades para enriquecer los currícula actuales.

5 http://wiki.secondlife.com/wiki/Second_Life_Education_Directory (consultada el 3 de septiembre de 2013)

6 http://www.simteach.com/wiki/index.php?title=Second_Life_Education_Wiki. (consultada el 4 de septiembre de 2013)

7 Iribas Rudín, Ana Eva (2008): Enseñanza virtual en Second Life: una opción online animada para las universidades y las artes. En IV Jornadas Campus Virtual, Madrid: UCM 


\section{Usos educativos de Second Life. El caso de UDIMA en la enseñanza con mundos virtuales y avatares.}

El entorno inmersivo de Second Life constituye, una plataforma de potenciación del aprendizaje, dado que reproduce metafóricamente escenarios judiciales, facilita y motiva el trabajo colaborativo en línea, al tiempo que contribuye a la adquisición de las competencias profesionales exigidas en la práctica jurídica del mundo real. Se discute así, el nuevo potencial tecnológico-pedagógico de la Web 2.0. en el marco de las estrategias docentes desarrolladas en la Universidad a Distancia de Madrid a fin de adecuar sus grados a las metodologías didácticas innovadoras que demanda el Espacio Europeo de Educación Superior (EEES) ${ }^{8}$.

Como señalan Daniel Livingstone y Jeremy Kemp (2006), la interfaz tridimensional resulta mucho más alegre para estudiantes que encuentran aburridas las plataformas de aprendizaje virtual (tales como WebCT y Moodle) porque sólo ofrecen entornos bidimensionales. Muchos estudiantes ya se manejan con soltura en entornos inmersivos tridimensionales, propios de los juegos de consolas y videojuegos online. Según estos autores, "la experiencia afectiva que los estudiantes disfrutan en estos entornos [...] no se puede comparar a las plataformas actuales de aprendizaje virtual".

Características y ventajas del uso de Second Life en la enseñanza Universitaria:

- La utilización de las nuevas tecnologías aplicadas a la educación favorecen un aprendizaje centrado en el estudiante.

- Una de las herramientas utilizadas en estos nuevos contextos de aprendizaje son los metaversos o mundos virtuales.

- Los usuarios pueden interactuar entre sí en tiempo real posibilitando entornos virtuales de aprendizaje mediante técnicas de role playing.

- En la UDIMA se crea un espacio virtual propio dentro de Second Life, en forma de isla, que utilizan los estudiantes durante el proceso de enseñanza-aprendizaje desarrollado en cada uno de los grados.

- Se ofrecen diferentes actividades a través de esta herramienta, desde practicar idiomas hasta realizar simulaciones de reuniones de trabajo.

Analizaremos a continuación, los objetivos propuestos por la UDIMA en los grados en Derecho, Psicología e Historia; grados en los que se aplica Second Life de una manera habitual y dinámica en diversas asignaturas de sus planes de estudio.

Objetivos Particulares (Grado en Derecho):

- Redactar escritos jurídicos relacionados con las asignaturas como el estímulo de la creatividad personal.

- Manejar el lenguaje técnico jurídico de Derecho Civil.

- Manejar bases de datos y sistemas informáticos para la búsqueda de información.

8 MONTERROSOSE. Y ESCUTIA R: Educación Inmersiva: enseñanza práctica del derecho en 3D. Revista Icono 14, 2011, Año 9 Vol 2, pp.84-100 ISSN 1697-8293 Madrid 
- Comprender por sí mismo las normas civiles y su aplicación práctica utilizando las normas procesales.

- Ejercitar la capacidad de argumentar y debatir acerca de las normas e instituciones civiles, en el seno de equipos o grupos de trabajo.

- Conocer las principales decisiones de la jurisprudencia en la aplicación de las normas e instituciones de Derecho Civil.

- Cuidar la forma y el fondo en las actuaciones del jurista.

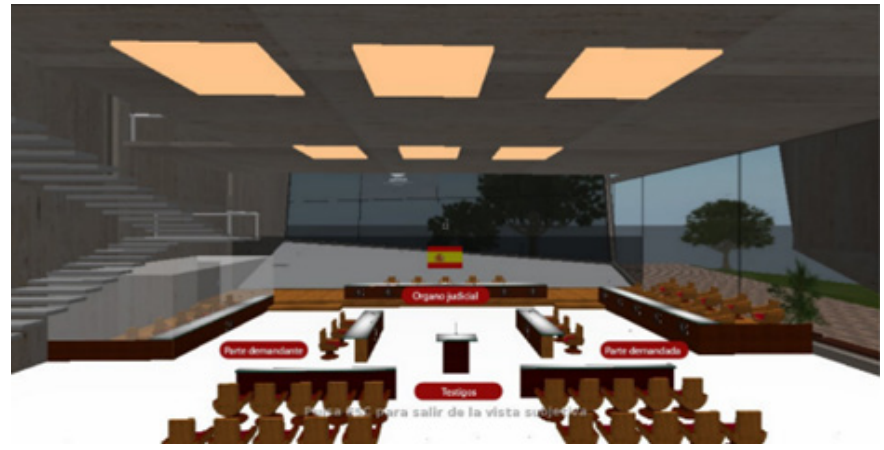

Aula de Juicio en Second Life. Fuente: udima.es ${ }^{9}$

Objetivos particulares (Grado en Historia):

- Completar el programa de actividades didácticas de la asignatura Historia Social y Política Contemporáneas con conceptos, obras y autores de la Historia de la pintura.

- Practicar procedimientos de comentario artístico ante una obra expuesta.

- Dar a conocer a los estudiantes la existencia de recursos culturales en Second Life.

- Realizar el trabajo en grupo de manera colaborativa.

- Aprender a trabajar online utilizando las tecnologías disponibles.

- Desarrollar competencias en materia de identificación y análisis de la obra de arte.

9 Aula 4 de la isla, http://slurl.com/secondlife/UDIMA/71/65/48/?title=Sala_de_Juicios_UDIMA). 


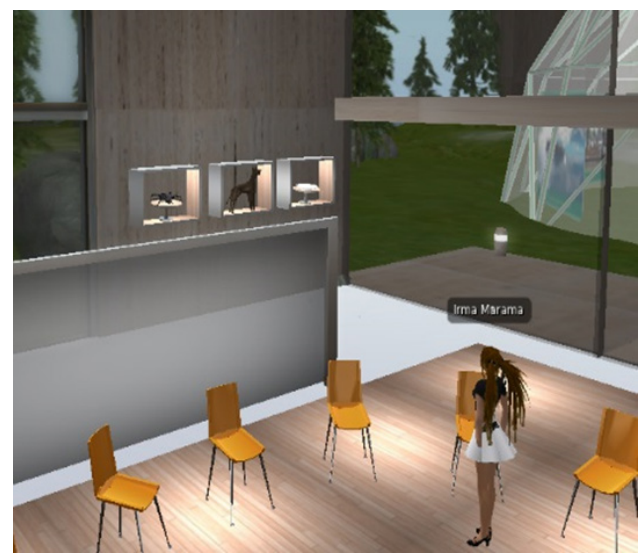

Avatar de alumna del grado en historia. Fuente: udima.es

Objetivos particulares (Grado en Psicología):

- Realizar prácticas sobre conocimientos adquiridos en la asignatura de Intervención y Tratamiento Psicológico.

- Desarrollar habilidades terapéuticas.

- Explorar posibilidades de Second Life como escenario para prácticas psicológicas.

- Realizar grabaciones de sesiones prácticas en Second Life.

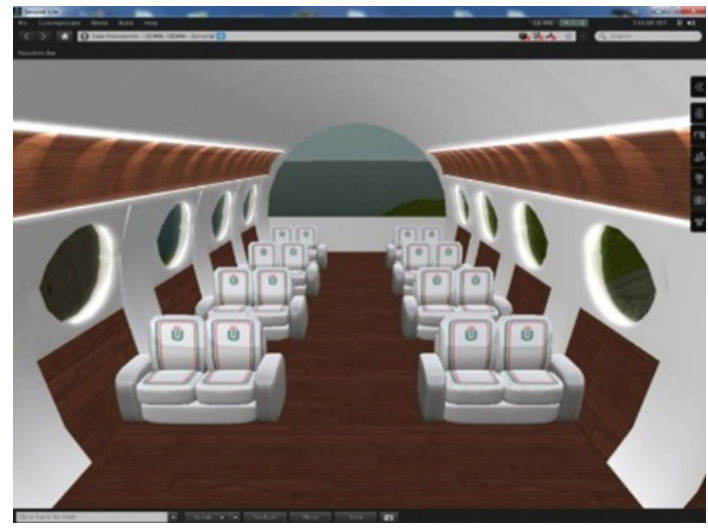

Unidad de psicología clínica de la asignatura de intervención y tratamiento psicológico de la UDIMA. Fuente: udima.es

Como vemos, el espacio virtual de UDIMA dentro de SL, en forma de isla, se ha diseñado como un espacio de interacción, comunicación y simulación de diferentes actividades de aprendizaje con los beneficios que los mundos virtuales proveen a la educación a distancia. Este tipo de actividades refuerzan y solidifican los conocimientos del estudiante a partir del método Learning-by-doing. 
Podemos, por tanto, concluir con las palabras del doctor Larry Johnson, CEO (Chief Executive Officer) de New Media Consortium (NMC), consorcio internacional sin ánimo de lucro dedicado al uso y exploración de nuevos medios audiovisuales y nuevas tecnologías en diferentes áreas, como la educación: "In a live videoconference, I could make a gesture and you'd see that, but we're still not together. We're not affecting each other's space. In Second Life, we... experience things together. ${ }^{10}$ ",

\section{Conclusiones}

Como hemos podido ver en el presente artículo y de acuerdo con las palabras de las doctoras Monterroso y Escutia, es ya una realidad que las nuevas tecnologías de la información y la comunicación ofrecen innumerables herramientas en diferentes entornos. Con ellas se puede llevar a cabo una estrategia educativa mucho más interactiva y adecuada para la adquisición de las competencias propias de los planes de estudio del EESS.

Actualmente se abre un mundo de posibilidades para la educación a distancia, lo que se conoce como entornos de realidad virtual, mundos virtuales o metaversos. Estos ambientes son simulaciones sobre el ordenador que tienen una interfaz en tercera dimensión para dar a sus usuarios la sensación de inmersión total en todas sus interacciones con el ambiente ${ }^{11}$.

Debido a las características y ventajas de los metaversos, estos tienen una aplicación directa en la educación, especialmente a distancia, ya que su uso pedagógico puede contribuir a disminuir las sensaciones de aislamiento, soledad e incomunicación que pueden experimentar los estudiantes a distancia durante su proceso de aprendizaje. Además, a través del uso pedagógico de los mundos virtuales, se pueden realizar actividades de aprendizaje participativas, constructivas y lo más parecidas posibles a cómo serían en el mundo real.

En este sentido, los entornos virtuales -en especial Second Life- ofrecen un servicio formativo de primera mano para los estudiantes y un medio útil en la enseñanza virtual universitaria ya que, además de potenciar la adquisición y transmisión de conocimientos, posibilita el aprendizaje a través de juegos de rol en los distintos ámbitos profesionales.

Estamos pues, ante una nueva forma de enseñanza y educación en la que el desarrollo y aplicación de estas nuevas herramientas en el aula, no se puede pasar por alto en la formación universitaria española del siglo XXI.

$10 \mathrm{http}: / /$ www.nmc.org/about/board-directors/larry-johnson-chief-executive-officer (consultada el 6 de septiembre de 2013)

$11 \mathrm{http}: / /$ www.udima.es/es/metaversos-master.html (consultada el 8 de septiembre de 2013) 


\section{Bibliografía}

Libros

CARPENTER, E., \& MCLUHAN, M. (1968). El aula sin muros. Cultura Popular. Barcelona

CARR, P., \& POND, G. (2007). Second Life: la guía definitiva a un nuevo mundo virtual. Random House Mondadori. Barcelona

CRARY, J. (2008). Suspensiones de la percepción. Atención, espectáculo y cultura moderna. Akal. Madrid

ECHEVERRÍA, J. (1999). Los señores del aire: Telépolis y el Tercer Entorno. Destino. Barcelona

LÉVY, P. (2007). Cibercultura. La cultura de la sociedad digital. Anthropos. Barcelona.

MURRAY, J. H. (1999). Hamlet en la Holocubierta. El futuro de la narrativa en el ciberespacio. Paidós. Barcelona.

Capítulos o artículos en libros o revistas en papel:

CARR, D. (2008). "Learning in Virtual Worlds for inclusion". En Selwyn, N. (Ed.), Education 2.0? A commentary by the Technology Enhaced Learning phase of the Teaching and Learning Research Programme, TLRP, pp. 17-22.

CASTRONOVA, E. (2001). "Virtual Worlds: A First-Hand Account of Market and Society on the Cyberian Frontier". En CESifo Working Paper No. 618.

DOWNES, R. (2008). "The Reality of Virtual Learning". DND Learn Conference, Cornwall Ontario January 30, 2008.

IRIBAS RUDÍN, Ana Eva (2008): Enseñanza virtual en Second Life: una opción online animada para las universidades y las artes. En IV Jornadas Campus Virtual, Madrid: UCM, pp.125-142.

MONTERROSOSE. Y ESCUTIA R: Educación Inmersiva: enseñanza práctica del derecho en 3D. Revista Icono 14, 2011, Año 9 Vol 2, pp.84-100 ISSN 1697-8293 Madrid.

STEPHENSON, N. (2005). Snow Crash. Barcelona: Gigamesh. ZYDA, M. (2005). "From Virtual Simulation to Virtual Reality to Games", IEEE Computer. Septiembre 2005, 27.

Artículos en publicaciones web:

CACIO, J. (2007). "Openness ant the Metaverse Singularity". En Kurzweil. Acceleraying Intelligence, November 7, 2007. http://www.kurzweilai.net/ openness-and-the-metaverse-singularity. [15 -09-2013].

HUDSBERGER, S. (2009). "Foreign language learn- ing in Second Life and the implications for resource provision in academic libraries". En arcadia@cambridge Publications: rethinking the role of the research library in a digital age. http://arcadiaproject.lib. cam.ac.uk. [28 -09-2013]. 
LIVINGSTONE, D., y KEMP, J. (mayo 2006): «Massively Multi-Learner: Recent Advances in 3D Social Environments». Computing and Information Systems Journal, vol. 10, n. ${ }^{\circ}$ 2. [13-09-2013] a través de http://www.cis.paisley.ac.uk/ research/journal/v 10n2/LivingstoneKemp.doc.

TEJADA José; NAVÍO, Antonio; RUIZ BUENO, Carmen (2007): La didáctica en un entorno virtual interuniversitario: experimentación de ECTS apoyados en TIC, Revista Pixel-Bit. Revista de Medios y Educación, 30. http://www.sav.us.es/pixelbit/pixelbit/articulos/n30/n30art/art303.htm. [1 -10-2013]

\section{Los autores}

Miguel Ángel Poveda Criado es Doctor en Periodismo (UCM), en Paz y Seguridad Nacional (UNED) y en Ciencias Políticas y Sociales (UTL). Profesor universitario de UDIMA, UFV, UE Madrid.

$\mathrm{M}^{\mathrm{a}}$ del Carmen Thous Tuset es Doctora en Periodismo (UCM) y Profesora universitaria de la Universidad Francisco de Vitoria de Madrid-España. 